\title{
Axicabtagene Ciloleucel CAR T-cell therapy for relapsed/refractory secondary CNS non-Hodgkin lymphoma: comparable outcomes and toxicities, but shorter remissions may warrant alternative consolidative strategies?
}

\author{
Sanaz Ghafouri $\mathbb{1 0}^{1} \cdot$ John Timmerman ${ }^{2} \cdot{\text { Sarah } \text { Larson }^{3} \cdot \text { Monica D. Mead }}^{4}$ \\ Received: 8 September 2020 / Accepted: 20 October 2020 / Published online: 10 November 2020 \\ (c) The Author(s), under exclusive licence to Springer Nature Limited 2020
}

\section{To the Editor:}

CAR T-cell therapy has changed the treatment landscape for patients with relapsed/refractory (R/R) B-cell nonHodgkin's lymphoma (NHL). The FDA approvals for axicabtagene and tisagenlecleucel in NHL were based on two phase 2 clinical trials, ZUMA-1 and JULIET, that resulted in overall response rates (ORR) of $82 \%$ with axicabtagene [1] and 52\% with tisagenlecleucel [2], respectively, in heavily pre-treated populations. Due to concerns that CNS involvement may increase risk and/or severity of CAR-Trelated neurotoxicity, the trials excluded patients with secondary CNS lymphoma. With this selected population, neurotoxicity was observed in $67 \%$ with axicabtagene and $39 \%$ with tisagenlecleucel [2, 3]. Limited data suggests CAR-T is feasible and does not result in excess toxicity in patients with active secondary CNS lymphoma, including single-institution experience of 8 patients who received tisagenlecleucel and a study from the US Lymphoma CAR$\mathrm{T}$ consortium that reported on 5 patients that received axicabtagene $[4,5]$. There remains a paucity of data regarding efficacy and toxicity profile of CAR-T, particularly for use

Sanaz Ghafouri

Snghafouri@mednet.ucla.edu

1 Department of Hematology Oncology, UCLA Medical Center, 757 Westwood Plaza, Los Angeles, CA 90095, USA

2 UCLA Lymphoma Program, Division of Hematology \& Oncology, Department of Medicine, UCLA Medical Center, 757 Westwood Plaza, Los Angeles, CA 90095, USA

3 Division of Hematology and Oncology, David Geffen School of Medicine at UCLA, UCLA Medical Center, 757 Westwood Plaza, Los Angeles, CA 90095, USA

4 Department of Hematology Oncology, UCLA Medical Center, 757 Westwood Plaza, Los Angeles, CA 90095, USA of axicabtagene, in patients with $\mathrm{R} / \mathrm{R}$ B-cell NHL with secondary CNS involvement.

We report the institutional experience at UCLA Medical Center from 5 patients treated with axicabtagene between October 2017 and January 2020 for R/R NHL with secondary CNS involvement. The UCLA IRB approved this clinical study, and all patients provided written informed consent for CAR-T. Patients' characteristics, toxicities and responses to CAR-T are shown in Table 1.

The median age at the time of CAR-T was 58.5 years (range, 28.3-76.4). This cohort includes three aggressive NHL histologies, one primary mediastinal B-cell lymphoma (PMBCL), two high-grade B-cell lymphomas with translocations involving $m y c$ and $b c l-2$ and/or $b c l-6$, and two activated B-cell (ABC) diffuse large B-cell lymphomas (DLBCL). The median number of prior therapies was 2.4 (range, 1-4). No patient underwent prior autologous or allogeneic hematopoietic stem cell transplant (HSCT). Median follow-up was 155.0 days (range, 86-208) after CAR-T.

At the time of CAR-T, three patients had concurrent systemic disease and all five patients had CNS involvement (two parenchymal, three parenchymal and leptomeningeal). All patients received CNS-directed therapy before lymphodepletion. Bridging therapy was administered in 3 patients.

Overall, axicabtagene was reasonably well-tolerated and there were no CAR-T-related deaths. Two patients developed grade 1 (Patient 4) and grade 2 (Patient 5) CRS, which responded to supportive care and tocilizumab, respectively. Notably, two patients developed ICANS; grade 3 in patient 2 without concurrent CRS and grade 4 in patient 5 with concurrent grade 2 CRS. Patient 2 developed expressive aphasia on day +7 that resolved with rapid steroid taper. Patient 5 developed status epilepticus on day +4 . Her symptoms resolved over 1 week with rapid steroid taper 


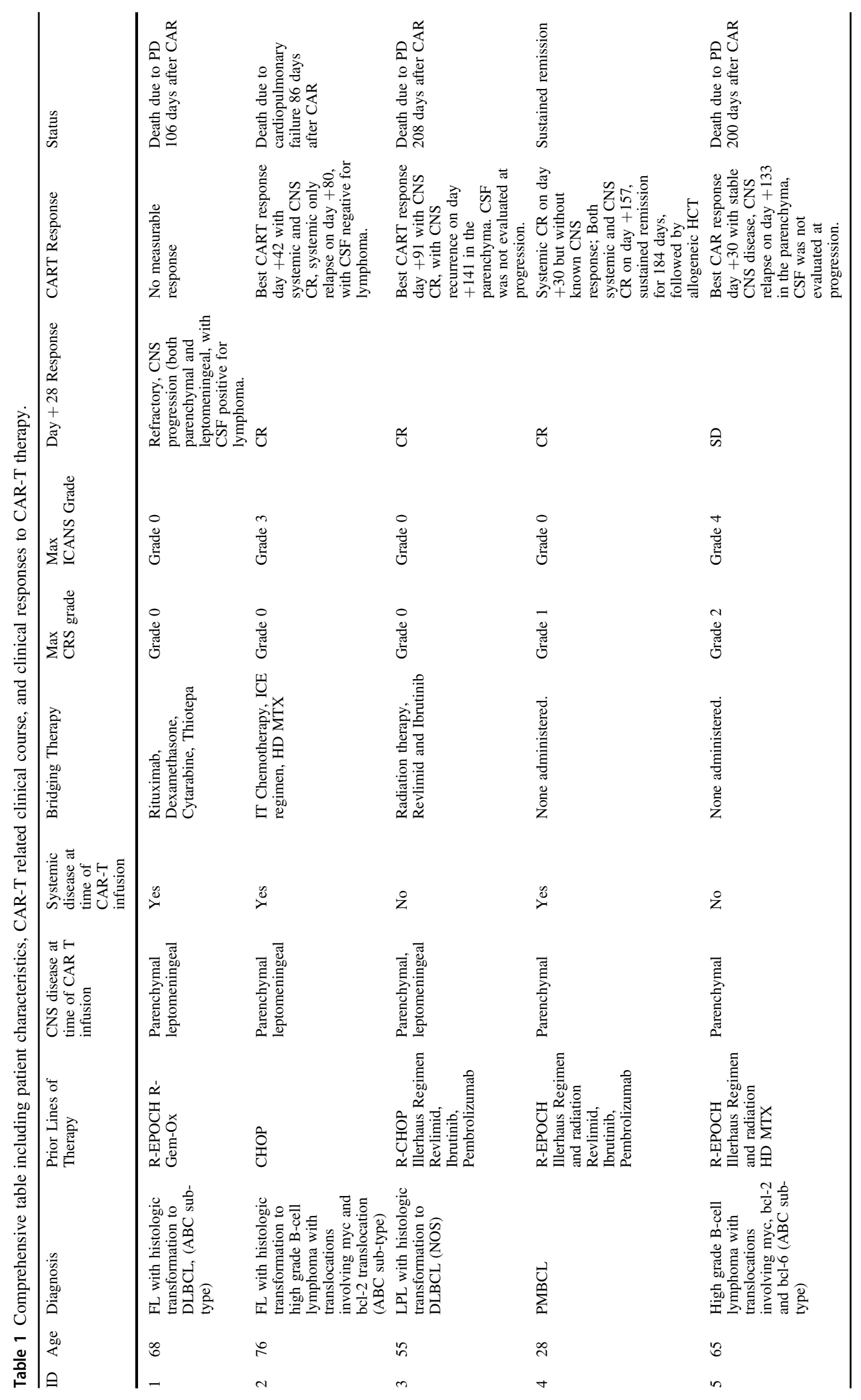


and dual anti-epileptics. She had no long-term neurologic sequelae.

Notably, 4 of the 5 patients (80\%) showed early response to CAR-T at day +28 (3 CRs; $1 \mathrm{SD}$ ). Responses were observed across all 3 aggressive NHL histologies in both systemic and CNS compartments. Three of the four responders developed progressive disease (PD) prior to 6month post-CAR-T disease reassessment. The remaining responder underwent an allogeneic-HSCT 184 days following CAR-T and remains alive at the time of submission. Of the four responders, the median PFS and OS was 134.2 days and 155.0 days (range, 86-208), respectively. The patient with CAR-T refractory disease (Patient 1) had no evidence for CRS or neurotoxicity.

R/R aggressive B-cell NHL with secondary CNS involvement is associated with a dismal prognosis. Despite efforts with chemotherapy, radiation and novel agents, response rates are low, and relapse is frequent. CAR-T has shown promising clinical benefit but the pivotal trials evaluating axicabtagene and tisagenlecleucel excluded patients with lymphomatous CNS involvement.

In this small series, the presence of secondary CNS lymphoma did not result in excess toxicity with axicabtagene. The $40 \%$ who developed ICANS were wellmanaged with a brief course of steroids and supportive care. These findings are similar to those reported for patients without lymphomatous CNS involvement in ZUMA-1, where $67 \%$ developed neurotoxicity, $32 \%$ of which was grade 3 or higher. Similarly, excess rates of CRS were not observed in our study population compared to that in the ZUMA-1 trial, and no patient in our series developed severe CRS [1].

While the development of CRS has been attributed to the indiscriminate activation and cytokine production from the immune system, the pathophysiology of ICANS is less well-described. Some studies demonstrate that disruption of the blood brain-barrier and increased vascular permeability leads to more severe neurotoxicities $[1,6,7]$. As experience with CAR-T has mounted, retrospective data have identified patient-related risk factors for developing ICANS including disease type, disease burden, treatment history and patient age. Our small series does not support secondary CNS lymphoma as an additional patient-related risk factor.

B-ALL literature describes the presence of CAR-T cells within the CNS persisting 6 months following CAR-T [8]. While the patient series by Frigault et al. evaluating tisagenlecleucel in patients with secondary CNS lymphoma did not directly measure CD19-directed CAR-T cells, biomarker analysis of the patients' CSF suggested CAR-T trafficking and expansion. Our study did not evaluate the CSF for inflammatory biomarkers or directly measure CAR$\mathrm{T}$ cells in the CSF. However, disease reduction in the CNS compartment in a subset of patients in our study, including a patient that did not have concurrent systemic disease, suggests axicel is trafficking to the CNS and undergoing expansion upon antigen recognition.

Axicabtagene resulted in an ORR of $80 \%$ at day +28 , and responses were observed across all 3 histologies, whether parenchymal and/or leptomeningeal CNS involvement. Of the 3 patients that achieved early CR, one patient had a leukemic and splenic relapse on day +82 and died before the CNS was restaged, one patient with isolated CNS relapse pre-CAR-T suffered a CNS-only relapse on day +141 and one patient maintained systemic and CNS CR by last assessment at day +184 and proceeded to an allogeneic-HSCT. The patient with SD had clinical benefit including reduction in headaches, imbalance and vision changes for $\sim 130$ days post CART infusion but had PD in the CNS-only on day +133 . The ORR of $80 \%$ at day +28 observed in our small series compares favorably with the day 28 ORR of $50 \%$ in a similar single-center experience with tisagenlecleucel and the best ORR of $60 \%$ in the subset of patients with active CNS lymphoma in the US Lymphoma CAR-T Consortium experience with axicel $[4,5]$. Putting our findings into context with results of ZUMA- 1 is limited by our low patient numbers and less comprehensive follow-up.

A strength of this study includes having a longer median follow-up, which showed that although $80 \%$ had an early response, the majority of responses were not durable. A consideration to infer is that patients may benefit from a novel CAR-T combination-based approach or a post- CAR$\mathrm{T}$ maintenance or consolidation strategy. Novel agents including BTK inhibitors, lenalidomide and checkpoint inhibitors have shown single-agent activity in R/R CNS lymphoma [9-11], making these agents a logical consideration for incorporation into CAR-T based strategies. Allogeneic-HSCT has not been widely employed for secondary CNS lymphoma due to the immune privilege of the CNS and concern for blunting of the graft-versuslymphoma effect. However, limited retrospective data describing 21 patients suggests this approach may result in long-term survival for a subset of patients [12]. Additional studies are needed to evaluate the incorporation of novel agents or the employment of a consolidative strategy to improve the outcomes of patients with $\mathrm{R} / \mathrm{R}$ NHL with secondary CNS involvement.

\section{Compliance with ethical standards}

Conflict of interest SG and MM have no conflict of interest. JT received research funding form BMS, Kite, ImmunGene. SL is consultant for BMS.

Publisher's note Springer Nature remains neutral with regard to jurisdictional claims in published maps and institutional affiliations. 


\section{References}

1. Neelapu SS, Tummala S, Kebriaei P, Wierda W, Gutierrez C, Locke FL, et al. Chimeric antigen receptor T-cell therapy assessment and management of toxicities. Nat Rev Clin Oncol. 2018;15:47-62.

2. Schuster SJ, Bishop MR, Tam CS, Waller EK, Borchmann P, McGuirk JP, et al. Tisagenlecleucel in adult relapsed or refractory diffuse large B-cell lymphoma. N Engl J Med. 2019;380:45-56.

3. Locke FL, Ghobadi A, Jacobson CA, Miklos DB, Lekakis LJ, Oluwole OO, et al. Long-term safety and activity of axicabtagene ciloleucel in refractory large B-cell lymphoma (ZUMA-1): a singlearm, multicentre, phase 1-2 trial. Lancet Oncol. 2019;20:31-42.

4. Frigault MJ, Dietrich J, Martinez-Lage M, Leick M, Choi BD, DeFilipp Z, et al. Tisagenlecleucel CAR T-cell therapy in secondary CNS lymphoma. Blood. 2019;134:860-6.

5. Bennani NN, Maurer MJ, Nastoupil LJ, Jain MD, Chavez JC, Cashen AF, et al. Experience with axicabtagene ciloleucel (Axi-cel) in patients with secondary cns involvement: results from the US lymphoma CAR T consortium. Blood. 2019;134:763.

6. Davila ML, Brentjens RJ. CD19-Targeted CAR T cells as novel cancer immunotherapy for relapsed or refractory B-cell acute lymphoblastic leukemia. Clin Adv Hematol Oncol. 2016; 14:802-8.

7. Gust J, Hay KA, Hanafi LA, Li D, Myerson D, Gonzalez-Cuyar LF, et al. Endothelial activation and blood-brain barrier disruption in neurotoxicity after adoptive immunotherapy with CD19 CAR-T Cells. Cancer Discov. 2017;7:1404-19.

8. Grupp SA, Kalos M, Barrett D, Aplenc R, Porter DL, Rheingold $\mathrm{SR}$, et al. Chimeric antigen receptor-modified $\mathrm{T}$ cells for acute lymphoid leukemia. N Engl J Med. 2013;368:1509-18.

9. Grommes C, Pastore A, Palaskas N, Tang SS, Campos C, Schartz $\mathrm{D}$, et al. Ibrutinib unmasks critical role of bruton tyrosine kinase in primary CNS lymphoma. Cancer Discov. 2017;7:1018-29.

10. Wilson WH, Young RM, Schmitz R, Yang Y, Pittaluga S, Wright G, et al. Targeting $\mathrm{B}$ cell receptor signaling with ibrutinib in diffuse large B cell lymphoma. Nat Med. 2015;21:922-6.

11. Rubenstein JL, Treseler PA, Stewart PJ. Regression of refractory intraocular large B-cell lymphoma with lenalidomide monotherapy. J Clin Oncol: Off J Am Soc Clin Oncol. 2011;29:e595-7.

12. Sterling C, Wagner-Johnston ND, Gladstone DE, Ambinder RF, Swinnen LJ, Imus PH. Allogenic stem cell transplantation for secondary CNS lymphoma: a retrospective review of 21 patients. Blood 2019;134:3342. 